\title{
eLS
}

\section{Plant Virus RNA Replication}

Alberto Carbonell*, Juan Antonio García, Carmen Simón-Mateo and Carmen Hernández

*Corresponding author: Alberto Carbonell (acarbonell@ibmcp.upv.es)

A22338

\section{Author Names and Affiliations}

Alberto Carbonell, Instituto de Biología Molecular y Celular de Plantas (CSIC-UPV), Campus UPV, Valencia, Spain

Juan Antonio García, Centro Nacional de Biotecnología (CSIC), Madrid, Spain

Carmen Simón-Mateo, Centro Nacional de Biotecnología (CSIC), Madrid, Spain

Carmen Hernández, Instituto de Biología Molecular y Celular de Plantas (CSIC-UPV), Campus UPV, Valencia, Spain

\section{*Advanced article}

\section{Article Contents}

- Introduction

- Replication cycles and sites of replication of plant RNA viruses

- Structure and dynamics of viral replication complexes

- Viral proteins involved in plant virus RNA replication

- Host proteins involved in plant virus RNA replication

- Functions of viral RNA in genome replication

- Concluding remarks

\begin{abstract}
Plant RNA viruses are obligate intracellular parasites with single-stranded (ss) or doublestranded RNA genome(s) generally encapsidated but rarely enveloped. For viruses with ssRNA genomes, the polarity of the infectious RNA (positive or negative) and the presence of one or more genomic RNA segments are the features that mostly determine the molecular mechanisms governing the replication process. RNA viruses cannot penetrate plant cell walls unaided, and must enter the cellular cytoplasm through mechanically-induced wounds or assisted by a
\end{abstract}


biological vector. After desencapsidation, their genome remains in the cytoplasm where it is translated, replicated, and encapsidated in a coupled manner. Replication occurs in large viral replication complexes (VRCs), tethered to modified membranes of cellular organelles and composed by the viral RNA templates and by viral and host proteins. Cis-acting elements located in viral RNA templates regulate the recognition by the virus-encoded RNA dependentRNA polymerase, and possibly contribute to VRC assembly and/or activation.

\section{Key words:}

Plant, RNA, virus, replication, host factor, RNA-dependent RNA polymerase, cis-acting; viral replication complex

\section{Key concepts:}

- The vast majority of plant RNA viruses have positive (mRNA sense) single-stranded RNA genome

- Some plant RNA viruses have negative single-stranded, or double-stranded RNA genome

- Replication is at the core of the infection cycle of plant RNA viruses and occurs solely through RNA intermediates of positive and negative polarities

- Replication of most plant RNA viruses takes place in viral replication complexes tethered to modified membranes of cytoplasmic organelles

- Viral replication complexes are dynamic, and contain the viral RNA, viral replication proteins and proteins from the host

- A viral RNA-dependent RNA polymerase is responsible for the synthesis of new genomic RNA using parental genome as template

- Viral proteins and co-opted host factors orchestrate the targeting of the genomic RNA to the membranes where replication takes place; they also contribute to the remodeling of the membranes that are used as scaffolds for tethering the viral replication complexes

- In addition to the role of co-opted host factors in controlling the biogenesis of replication organelles and the composition, assembly and/or activity of the viral replication complex, they can post-translationally modify viral proteins to regulate replication 
- Primary sequence and structure elements of viral RNAs regulate template selection, initiation of the synthesis of complementary RNA strands and the assembly and activation of viral replication complexes

\section{Introduction}

Plant viruses are obligate intracellular parasites with deoxyribonucleic acid (DNA) or ribonucleic acid (RNA) genome that require host's molecular machinery to complete their life cycle. The viral genome is generally encapsidated by one or more viral coat proteins (CPs) to form a virion that may have distinct architectures and that sometimes can be enveloped. RNA viruses can be divided into three classes on the basis of whether their virions package the genome as mRNA sense [positive (+) strand] single-stranded (ss) RNA, antisense [negative () strand] ssRNA or double-stranded (ds) RNA. In all three classes, the viral genome can be formed by either a single molecule (non-segmented genome) or by two or more molecules (segmented genome), each of which is named as genomic RNA (gRNA). Some of these features are listed for the type member of each plant RNA virus genus in Table 1. See also: Capsid-Less RNA viruses (DOI: 10.1002/9780470015902.a0023269); Multi-component Plant Viruses (DOI: 10.1002/9780470015902.a0024783); RNA virus genomes (DOI: 10.1002/9780470015902.a0001488.pub2); Virus Classification and Nomenclature (DOI: $\quad$ 10.1002/9780470015902.a0000440.pub3); $\quad$ Viruses $\quad$ (DOI: 10.1002/9780470015902.a0000441.pub3); Virus $\quad$ structure 10.1002/9780470015902.a0000439.pub2)

Plant viruses cannot penetrate plant cell walls unaided, thus most of them are introduced into the cell by a vector organism (usually insects, nematodes or fungi) that feeds on the plant or through mechanically-induced wounds. Multiplication of most RNA viruses occurs in the host cell's cytoplasm and follows a series of highly regulated and coordinated events that ultimately leads to production of newly formed virions that may then spread to nearby cells and be transmitted to other plants. The replication of the viral genome is a central step in the virus multiplication cycle and, in the case of RNA viruses, proceeds solely through RNA intermediates and takes place in large viral replication complexes (VRCs) -containing the viral RNA, viral replication proteins and proteins from the host- anchored to modified membranes of diverse cellular organelles. Distinct types of cis-acting elements located in viral RNAs dictate/regulate template selection, initiation of the synthesis of complementary RNA strands and VRC assembly and activation. See also: Replication of Viruses Infecting Eukaryotes (DOI: 
10.1002/9780470015902.a0021993); RNA Plant and Animal Virus Replication (DOI: 10.1038/npg.els.0001086); Virus Replication (DOI: 10.1002/9780470015902.a0000438.pub2)

\section{Replication cycles and sites of replication of plant RNA viruses}

Once a plant RNA virus enters a cell, desencapsidation occurs and the virus genome is released to the cytoplasm where, with the only exception of members of genus Nucleorhabdovirus (see below), all steps of the replication cycle are completed. The molecular events underlying replication depend on the type of RNA genome. For $(+)$ RNA viruses, the most abundant class of plant RNA viruses, the gRNA is first translated into the different viral proteins, including the RNA dependent RNA polymerase $(\mathrm{RdRp})$ that is the responsible for the copy of $(+)$ strands into (-) strands. The latter ones serve as templates for generating multiple $(+)$ strands that are translated, replicated or assembled into virions that may spread throughout the plant and/or be transmitted to new hosts (Figure 1). In the case of (-) RNA viruses, the viral RdRp is packaged along the gRNA within the virion so that it can initiate synthesis of $(+)$ strands complementary to the infectious (-) gRNA. The (+) strands are then translated into viral proteins or used as templates for the generation of new (-) strands that can either serve as templates for additional replication rounds or be assembled into progeny virions (Figure 1). Regarding dsRNA viruses, the viral RdRp is also incorporated into the virions where it synthesizes (+) RNA strands that are extruded from the particle. Such (+) RNA strands are first translated to produce, among others, the RdRp and the CP and then packaged to form immature virions that develop by synthesizing (-) RNA strands. In this way, while the dsRNA genome is being produced, it is protected from host antiviral systems such as RNA silencing that detect and inactivate dsRNA molecules (Figure 1). See also: Replication of Viruses Infecting Eukaryotes (DOI: 10.1002/9780470015902.a0021993); RNA Plant and Animal Virus Replication (DOI: 10.1038/npg.els.0001086); RNA virus genomes (DOI: 10.1002/9780470015902.a0001488.pub2); Virus Classification and Nomenclature (DOI: 10.1002/9780470015902.a0000440.pub3); Virus Replication (DOI: 10.1002/9780470015902.a0000438.pub2)

Replication occurs in membranes of cytoplasmic organelles such as the endoplasmic reticulum (ER), mitochondrias, vacuoles, peroxisomes or chloroplasts, except for (-) RNA viruses of the genus Nucleorhabdovirus that replicate in the nucleus in association with nuclear membranes (Table 2). Certain plant RNA viruses can exploit alternative subcellular membranes for their replication in the absence of their primary membrane, as shown for 
tombusviruses Tomato bushy stunt virus (TBSV) and Cymbidium ringspot virus (CymRSV) or potyvirus Turnip mosaic virus (TuMV) (Xu and Nagy, 2014). In this review we describe essentially key aspects of $(+)$ RNA virus replication, as the lack of reverse genetics approaches for (-) RNA and dsRNA viruses has limited the study of these two classes of viral agents. See also: Cell membrane features (DOI: 10.1002/9780470015902.a0001261.pub3); Nuclear membranes (DOI: 10.1002/9780470015902.a0026032); Plant chloroplasts and other plastids (DOI: 10.1002/9780470015902.a0001678.pub2); Plant endoplasmic reticulum (DOI: 10.1002/9780470015902.a0001673.pub2; Plant Golgi apparatus (DOI: 10.1002/9780470015902.a0001674.pub2); Plant mitochondria (DOI: 10.1002/9780470015902.a0001680.pub2); Plant peroxisomes and glyoxysomes (DOI: 10.1002/9780470015902.a0001677.pub2); Plant vacuoles (DOI: 10.1002/9780470015902.a0001675.pub2); Rhabdoviruses $\quad$ (DOI: 10.1002/9780470015902.a0001085.pub3)

\section{Structure and dynamics of viral replication complexes}

For plant RNA viruses, the architecture of Brome mosaic virus (BMV) VRCs is the best known. BMV replication occurs in vesicles -known as spherules- derived from ER invaginations and with an open neck that connects its interior with the cytoplasm (Diaz and Wang, 2014). It has been proposed that these spherules may protect BMV VRCs (as do capsids in retroviruses or dsRNA viruses) from cellular defenses, and that similar structures may be involved in the replication of other ssRNA viruses (den Boon et al., 2010; Nagy and Pogany, 2012). Interestingly, BMV replication can also occur in very different membranous structures. By modulating the relative levels and interactions of BMV replication factors it is possible to produce multilayered stacks of ER-derived appressed double membranes that support RNA replication as efficiently as the spherules (Diaz and Wang, 2014).

Importantly, the membranous structures where VRCs are assembled are not static during the infection process. For instance, multiple replication sites that are distributed randomly throughout the cytoplasm at early stages of Cowpea mosaic virus (CPMV) infection rapidly coalesce into a large body located in the center or the cell, often in the perinuclear region (Carette et al., 2002). Similar perinuclear structures have also been observed in infections by other RNA viruses (Heinlein, 2015). While those from secoviruses CPMV and Grapevine fanleaf virus (GFLV) seem to derive from ER membranes, those from potexvirus Potato virus $X$ (PVX) and potyvirus Turnip mosaic virus (TuMV) also contain elements of the Golgi 
apparatus, and in the case of TuMV, of chloroplasts. TuMV vesicles move along the perinuclear body and the nuclear periphery, and traffic through the actomyosin motility system from the ER to the chloroplasts, where they amalgamate and induce chloroplast membrane invaginations. Similarly, Alfalfa mosaic virus (AMV) VRCs are assembled in multivesicular bodies before their recruitment to the vacuolar membrane or tonoplast. Also, it has been observed that the intracellular trafficking of Tobacco mosaic virus (TMV) VRCs along microfilaments is required for the cell to cell spread of the infection (Heinlein, 2015). See also: Secoviridae: a family of plant picorna-like viruses with monopartite or bipartite genomes (DOI: 10.1002/9780470015902.a0000764.pub3)

\section{Viral proteins involved in plant virus RNA replication}

All plant RNA viruses encode a viral RdRp that is the responsible for the synthesis of new gRNA using parental gRNA as template. In the most simple plant RNA viruses, viral proteins involved in replication are restricted to such $\operatorname{RdRp}$ and to a second protein with variable functions depending on the virus species. For example, the tobamovirus TMV produces a $180 \mathrm{~K}$ protein, with a RNA polymerase domain, and a $130 \mathrm{~K}$ protein that methylates guanosine $-5^{\prime}-$ triphosphate (GTP) and transfers the resulting $\mathrm{m}^{7} \mathrm{GTP}$ to the $5^{\prime}$ end of the nascent RNA molecule to generate a cap structure (Ishibashi et al., 2012). Besides its role as methyltransferase, this protein also acts as RNA helicase hydrolyzing nucleoside triphosphates (NTPs) and using the resulting energy to unwind the two strands of the dsRNA replication intermediates (Goregaoker and Culver, 2003; Nishikiori et al., 2012). Moreover, the interaction of $130 \mathrm{~K}$ with host proteins is critical for membrane targeting of the ribonucleic complexes formed by viral RNAs and replication proteins that are assembled co-translationally and that ultimately constitute the functional VRCs (Ishibashi et al., 2012).

The replication of members of the Bromoviridae family also requires only two viral proteins: 1a protein, with a methyltransferase and a NTPase/helicase domain similar to those of TMV $130 \mathrm{~K}$, and 2a protein, containing a central RNA polymerase domain and a N-terminal domain that interacts with the helicase domain of $1 \mathrm{a}$. BMV 1a has been shown to play a critical role also in VRC assembly. In particular, BMV 1a localizes in ER membranes, where it oligomerizes, induces the formation of invaginations and recruits both the $2 \mathrm{a}$ RNA polymerase and the template RNAs to these compartments where replication takes place (Diaz and Wang, 2014). See also: Bromoviridae and allies (DOI: 10.1002/9780470015902.a0000745.pub3) 
It seems that a general characteristic of plant ( + ) RNA viruses is to express a protein to induce precise modifications in cellular membranes and to target there the viral factors that are required to assemble functional VRCs. Hence, p33 from tombusviruses induces the formation of spherules that are similar to those induced by BMV 1a, and directs to them the RNA template and the p92 RNA polymerase (Stork et al., 2011). Protein p33 has also RNA chaperone activity, which might assist in both the assembly and activity of VRCs. Red clover necrotic mosaic virus (RCNMV), a dianthovirus also from the Tombusviridae family, expresses two proteins, p27 and p88, involved in RNA replication. Protein p27 has a strong affinity for ER membranes, interacts with itself and with p88 as well as with the viral RNA and with several host proteins to facilitate the VRC assembly (Hyodo et al., 2014). In tymovirus Turnip yellow mosaic virus (TYMV) infections, the $140 \mathrm{~K}$ protein, containing methyltransferase, proteinase and NTPase/helicase domains, recruits the $66 \mathrm{~K}$ RNA polymerase to the chloroplast membrane (Prod'homme et al., 2003). See also: Tombusviridae (DOI: 10.1002/9780470015902.a0000756.pub3)

Other plant RNA viruses with more complex genomes such as closteroviruses or potyviruses devote a larger number of proteins to their replication. Genetic studies have revealed that the majority of potyviral gene products participate either directly or indirectly in replication. However, while the roles that certain potyviral proteins such as the RNA polymerase NIb or the NTPase/helicase CI play in replication are well known, those of other proteins such as P3 remain to be ascertained. An example of potyviral protein with known role in replication is $6 \mathrm{~K}_{2}$, a small hydrophobic peptide that anchors the VRC to the membrane. Despite this protein can localize by itself to the ER and induce the formation of membranous vesicles, it seems that the partially processed $6 \mathrm{~K}_{2}$-VPg-Pro product is actually the skeleton upon the VRC is built (Heinlein, 2015). Moreover, this protein contains the protease domain of NIa and the multifunctional protein VPg, which binds covalently to the $5^{\prime}$ end of the potyviral gRNA and is urilydated by the NIb viral RNA polymerase (Puustinen and Mäkinen, 2004; Anindya et al., 2005). The resulting product is thought to be used as initiator of viral RNA replication. See also: Closteroviridae (DOI: 10.1002/9780470015902.a0000747.pub3); Potyviridae (DOI: 10.1002/9780470015902.a0000755.pub3)

\section{Host proteins involved in plant virus RNA replication}

The relatively small size and consequently limited coding capacity of viral genomes could explain why plant RNA viruses must hijack certain cellular pathways, co-opt host factors (HFs) 
and manipulate cellular structures to be able to complete all the steps of their life cycle, including genome replication. The search of co-opted HFs involved in virus replication has been the subject of intense research and has been addressed through different approaches including in vitro replication assays, reverse and direct genetics and intracellular localization studies. In addition, the yeast Saccharomyces cerevisiae has been reported to sustain replication of some plant (+) RNA viruses such as BMV or TBSV. Genome-wide screenings in this model organism have been extremely useful to identify HFs participating in the replication of these viruses (Galao et al., 2007; Nagy et al., 2014). Identified HFs include RNA-binding proteins, chaperones, translation factors or proteins involved in membrane trafficking or synthesis, among others (Table 3). Generally, co-opted HFs control the biogenesis of replication organelles, the composition, assembly or activity of the VRCs, or mediate post-translational modifications of viral replication proteins. The best studied HFs regulating these processes are described below and are listed in Table 3 together with other factors that are not mentioned in the text due to space limitations.

\section{Host factors involved in the biogenesis of viral replication organelles}

Increasing evidence indicates that viral proteins and co-opted HFs orchestrate the protein targeting to specific membranes as well as the remodeling of certain cellular membranes used as scaffolds for tethering the VRCs. A first group of HFs involved in the biogenesis of replication organelles includes proteins participating in lipid metabolism and membrane organization. Indeed, membrane rearrangements are necessary for the formation of VRCs, and require the modification of the lipid composition of the membrane. For example, OLE1, a $\Delta 9$ fatty acid desaturase, is important for BMV replication because it regulates the fluidity or plasticity of the membrane at the replication site (Diaz and Wang, 2014). In contrast, BMV replication is severely inhibited when ACB1-encoded acyl-CoA binding protein (ACBP), a critical enzyme maintaining lipid homeostasis, is mutated (Diaz and Wang, 2014). Other genetic studies in yeast have identified several host genes involved in lipid, fatty acid or sterol biosynthesis/metabolism that affect TBSV replication (Wang, 2015). See also: Lipids (DOI: 10.1002/9780470015902.a0000711.pub3); Membrane lipid biosynthesis (DOI: 10.1002/9780470015902.a0001391.pub2)

It has also been observed that viruses use components of the cellular secretory pathway for the organization of the membranous compartments where VRCs are assembled. For instance, DOA4 and BRO1, two components of the multivesicular body secretory pathway, are important for BMV replication because they are required for the ubiquitination and 
proteasome-dependent activation of transcription factors MGA2 and SPT23 regulating several lipid metabolism genes, including OLE1 (Diaz and Wang, 2014). Another component of the cellular secretory machinery that is important for viral replication is ARF1, a small GTPase involved in COPI vesicle formation in the Golgi apparatus. Its interaction with RCNMV p27 redirects ARF1 to the ER membranes where it participates in VRC formation (Hyodo et al., 2013). Similarly, ARL8 GTPase interacts with Tomato mosaic virus (ToMV) 130K, and, together with TOM1 and TOM3, contributes to $130 \mathrm{~K}$ targeting to ER membranes and to activate the VRC (Ishibashi et al., 2012). PEX19, required for the synthesis of peroxisome membranes, also acts as a cellular transporter for directing TBSV p33 to the peroxisomal membrane to initiate TBSV VRC assembly (Pathak et al., 2008). Finally, another function of certain secretory proteins is to induce membrane bending in order to facilitate the formation of spherules where viral RNA is synthesized. This is apparently the case of ESCRT (endosomal sorting complexes required for transport) and RHP (reticulon homology proteins) factors in TBSV and BMV infections (Wang, 2015). See also: Protein secretory pathways (DOI: 10.1038/npg.els.0005723)

\section{Host factors involved in the assembly and activity of viral replication complexes}

Host proteins with chaperone activity (e.g. heat-shock proteins, HSPs) or RNA binding capacity have also been found as a part of VRCs (Table 3) (Nagy et al., 2011; Verchot, 2012). In tombusvirus infections, HSP70 recruitment is required for the insertion of VRC components in the corresponding intracellular membranes, as well as for their correct assembly in these structures. HSP70 is also necessary for the replication of ToMV, TMV, TuMV and RCNMV (Verchot, 2012). In Potato virus A (PVA) infections, HSP70 requires its co-chaperone CPIP to regulate the amount of CP in VRCs at early stages of the infection (Hafren et al., 2010). Another cellular chaperone involved in replication of (+) RNA viruses is HSP90, which stimulates Bamboo mosaic virus (BaMV) replication through its interaction with both the 3' untranslated region (UTR) of BMV gRNA and RdRp (Huang et al., 2012). Indeed, both HSP70 and HSP90 participate in RCNMV replication and, despite both of them interact with p27, they regulate different steps of VRC assembly (Mine et al., 2012). Another HSP, HSP40, contributes to the folding of BMV p2a and to its assembly into active VRCs (Tomita et al., 2003). In contrast to HSPs, other cellular proteins with chaperone activity can inhibit viral replication, as observed for CPR1 and CPR7 that interact with TBSV replication proteins (Mendu et al., 2010; Lin et al., 2012). 
Other HFs have a more direct role in gRNA synthesis. For instance, eukaryotic translation elongation factors $1 \mathrm{~A}(\mathrm{eEF} 1 \mathrm{~A})$ and/or 1B (eEF1B) interact with the RdRp of several RNA viruses and, therefore, probably participate in regulating viral gRNA synthesis (Hwang et al., 2013). In TBSV infection, eEF1A is present in the VRC and interacts with $\mathrm{p} 33$ and $\mathrm{p} 92$ as well as with a cis-acting RNA element present at the 3'end of TBSV RNA (Li et al., 2009). eEF1A assists the recruitment of the viral RNA and the VRC assembly, and promotes (-) RNA synthesis (Li et al., 2010). Another co-opted HF involved in virus replication is GAPDH (glyceraldehyde-3-phosphate dehydrogenase), which has opposite effects on viral replication depending on the virus. In TBSV infection, GAPDH is recruited by TBSV p92 to the VRC, binds an AU pentamer sequence near the 3' end of (-) RNA and regulates asymmetry in the synthesis of (+) and (-) viral RNAs by anchoring (-) strands inside the VCR while the (+) RNA progeny is released to the cytosol (Wang and Nagy, 2008; Huang and Nagy, 2011). In contrast, GAPDH has more affinity for BaMV (+) RNA strands and has an inhibitory effect on viral replication (Prasanth et al., 2011). On the other hand, viruses with small genomes such as those of the Tombusviridae family do not encode helicase-like proteins. Thus, it is assumed that they must recruit host helicases to assist in their replication. In line with this view, distinct DEADbox RNA helicases (DED1 and DBP2 from yeast, and AtRH20, AtRH2 and AtRH5 from Arabidopsis thaliana) have been found to be involved directly in TBSV RNA replication. These HFs seem to bind and unwind local secondary structures at the 3 'end of the viral (-) RNA thus facilitating the synthesis of (+) RNA (Kovalev and Nagy, 2014).

\section{Host factors involved in the post-translational modification of viral proteins}

Different events of the virus life cycle (e.g. translation, assembly, spread to neighboring cells) are coupled to viral RNA replication. Therefore, it is not surprising that viral proteins undergo post-translational modifications to increase their functional diversity. Phosphorylation of certain viral proteins negatively regulates virus replication as described for RNA polymerase 2a from CMV that is phosphorylated by CIPK12 (calcineurin B-like calcium sensor-interacting protein kinase 12) at the late infection stages in Nicotiana tabacum (Kim et al., 2002). Another example of viral proteins undergoing phosphorylation are the replication-related proteins $66 \mathrm{~K}$ and p33 from TYMV and Cucumber necrosis virus (CNV), respectively (Wang, 2015). In the case of TBSV, the overexpression of protein kinase $\mathrm{C} 1$ (PKC1) identified in a genome-wide overexpression proteome screen in yeast inhibits TBSV replication (Shah Nawaz-ul-Rehman et al., 2012). 
Ubiquitination of viral proteins also appears to play an important role in regulating viral replication, as observed for TBSV p33 whose balanced ubiquitination by E2 ubiquitinconjugating enzyme CDC34 is required for its interaction with HFs in yeast (Barajas and Nagy, 2010). RSP5, an E3 ubiquitin ligase, interacts with TBSV p33 and p92, and inhibits TBSV replication in yeast when overexpressed (Barajas et al., 2009). Interestingly, host-dependent ubiquitination of TYMV $66 \mathrm{~K}$ can be counteracted by viral $98 \mathrm{~K}$ RNA polymerase that deubiquitinates $66 \mathrm{~K}$ to regulate viral replication (Camborde et al., 2010; Alcaide-Loridan and Jupin, 2012; Chenon et al., 2012). More recently, other post-translational modifications of viral or host proteins affecting viral replication have been described. This is the case of the SUMOylation of TuMV NIb RNA polymerase by SUMO-conjugating E2 enzyme SCE1 (Xiong and Wang, 2013), or the methylation of host eEF1A by the mETTL10-like SEE1 methyltransferase that inhibits TBSV replication in yeast and plants (Li et al., 2014). See also: Proteins: postsynthetic modification - Function and physical analysis (DOI: 10.1002/9780470015902.a0000551.pub2); SUMOylation

(DOI: 10.1002/9780470015902.a0021849.pub2)

\section{Functions of viral RNA in genome replication}

The viral RNA performs both template- and non-template functions during replication. The latter ones include its recruitment to the replication site and the assembly of a functional VRC. A variety of RNA elements (most of which act in cis) regulating these processes have been identified and characterized (Newburn and White, 2015). They are present either in the 3' UTR, internal regions, or the 5' UTR of viral RNAs, and include linear nucleotide sequences, RNA segments that may fold into characteristic secondary structures (e.g. stem-loops, SLs), or others adopting high order structures that may also participate in medium- or long-range RNA:RNA interactions. Some of the best-studied examples of viral cis-acting RNA elements involved in genome replication of plant RNA viruses are described below.

The replication of tombusviruses, exemplified by TBSV, has been intensively studied in the last years. This work has led to the identification of the RNA motifs that act as core promoters for (+) and (-) strand synthesis initiation (Pathak et al., 2011). In the $3^{\prime}$ UTR of TBSV (+) gRNA, a SL structure (SL1) and the adjacent 3 '-terminal nucleotides constitute the core promoter for (-) strand synthesis, whose activity is negatively regulated by its interaction with an internal loop in a proximal SL (SL3) (Figure 2a). The presence in other tombusvirids of similar short-range interactions regulating core promoter activity suggests that this is a well- 
conserved mechanism for the modulation of viral replication. The core promoter for $(+)$ strand synthesis corresponds to the 3 '-terminal $11 \mathrm{nt}$ of TBSV (-) strand of gRNA, and its activity is positively regulated by elements that are located on the same (-) strand molecule. The existence of elements in the (+) and (-) gRNA that act as repressors and enhancers, respectively, of the synthesis of the corresponding complementary strands most likely accounts for the strand asymmetry that characterizes the replication process in $(+)$ RNA viruses, with $(+)$ strands being produced in a large excess over (-) strands.

Another important RNA element for TBSV replication is located in the 5' UTR of the gRNA (Figure 2a). The sequence and the secondary and tertiary structures of this element are important for the accumulation of TBSV RNA of both polarities (Ray et al., 2004). In addition, another SL structure, known as RII(+)SL, that contains a critical CC mismatch and that is located in the p92/RdRp coding region of TBSV RNA (Figure 2a), is recognized by the viral protein p33, a recognition that seems essential for recruitment of the viral template for replication. The interactions between RII(+)-SL and the 3' terminal SLs, and between an upstream (UL) and a downstream (DL) linker, result in the formation of a bipartite RNA platform that assists the VRC assembly (Figure 2a) (Pathak et al., 2011; Pathak et al., 2012). In the case of the dianthovirus RCNMV, a Tombusviridae member with bipartite RNA genome, both gRNAs contain RNA elements important for replication (Pathak et al., 2011). RNA1 contains two SLs (SLDE and SLF) that form the core promoter for (-) strand synthesis (Figure 2b). RNA2 also contains two SLs (SL11 and SL13) that are the core promoter for (-) strand synthesis of this genomic segment (Figure 2b). In addition, RNA2 includes at least two other RNA elements that are critical for (-) strand synthesis: a Y-shape RNA element (YRE) located in the 3' UTR which routes RNA2 to the ER membrane for VRC assembly, and an element (TA) in the coding region of $\mathrm{p} 33$ that might interact with host proteins required for viral replication (Pathak et al., 2011) (Figure 2b). See also: Tombusviridae (DOI: 10.1002/9780470015902.a0000756.pub3)

Another extensively studied virus in terms of replication is BMV. The three BMV gRNAs fold in a tRNA-like (TSL) structure at their 3'-terminal regions, with a SL (SLC) being critical for VRC assembly (Figure 2c) (Wang, 2015). Moreover, a SL RNA motif named B-box is present in the 5' UTR of RNA1 and RNA2 as well as in the intercistronic region of RNA3 (Figure 2c). In all cases these B-box motifs are critical for the interaction with BMV 1a protein and subsequent recruitment of the viral RNAs for replication (Wang, 2015). Another SL structure at the $3^{\prime}$ end of RNA2 (-) strand has been identified as the core promoter for $(+)$ strand 
production (Sivakumaran et al., 1999). See also: Bromoviridae and allies (DOI: 10.1002/9780470015902.a0000745.pub3)

\section{Concluding remarks}

Replication is at the core of the infection cycle of plant RNA viruses. It represents the complex process by which RNA viruses copy and package their genome to infect new cells and, hence, ensure their survival. During the past years, our knowledge on how RNA viruses replicate in plant cells has increased significantly. In particular, for $(+)$ RNA viruses, this is explained by i) the availability of suitable reverse genetic approaches due to the relative ease to construct infectious cDNA clones that allow to modify virus genomes and evaluate in planta the effect of these modifications on virus biology, and ii) the ability of some (+) RNA viruses to replicate in yeast, an alternative experimental host with a well-annotated genome that has facilitated genome-wide searches for HFs involved in virus replication. The future development of similar approaches for studying (-) RNA and dsRNA viruses is thus critical to better understand how these two classes of virus replicate. More generally, a deeper understanding of virus replication should facilitate the development of novel strategies for inducing more effective antiviral resistance in plants.

\section{Acknowledgements}

Work in the authors' laboratories is supported by grants BFU2012-36095 BIO2013-49053$\mathrm{R}$ and Plant KBBE PCIN-2013-056 from the Ministerio de Economía y Competitividad (MINECO, Spain), and from the European Union's Horizon 2020 research and innovation programme under the Marie Sklodowska-Curie grant agreement No 655841.

\section{References}

Alcaide-Loridan C and Jupin I (2012) Ubiquitin and plant viruses, let's play together! Plant Physiology 160: 72-82.

Anindya R, Chittori S and Savithri HS (2005) Tyrosine 66 of Pepper vein banding virus genome-linked protein is uridylylated by RNA-dependent RNA polymerase. Virology 336: $154-162$.

Barajas D, Jiang Y and Nagy PD (2009) A unique role for the host ESCRT proteins in replication of Tomato bushy stunt virus. PLoS Pathogens 5: e1000705. 
Barajas D and Nagy PD (2010) Ubiquitination of tombusvirus p33 replication protein plays a role in virus replication and binding to the host Vps23p ESCRT protein. Virology 397: $358-368$.

Camborde L, Planchais S, Tournier V, et al. (2010) The ubiquitin-proteasome system regulates the accumulation of Turnip yellow mosaic virus RNA-dependent RNA polymerase during viral infection. The Plant Cell 22: 3142-3152.

Carette JE, Guhl K, Wellink J and Van Kammen A (2002) Coalescence of the sites of cowpea mosaic virus RNA replication into a cytopathic structure. Journal of Virology 76: 6235-6243.

Chenon M, Camborde L, Cheminant S and Jupin I (2012) A viral deubiquitylating enzyme targets viral RNA-dependent RNA polymerase and affects viral infectivity. $E M B O$ Journal 31: 741-753.

den Boon JA, Diaz A and Ahlquist P (2010) Cytoplasmic viral replication complexes. Cell Host Microbe 8: 77-85.

Diaz A and Wang X (2014) Bromovirus-induced remodeling of host membranes during viral RNA replication. Current Opinion in Virology 9: 104-110.

Galao RP, Scheller N, Alves-Rodrigues I, et al. (2007) Saccharomyces cerevisiae: a versatile eukaryotic system in virology. Microbial Cell Factories 6: 32.

Goregaoker SP and Culver JN (2003) Oligomerization and activity of the helicase domain of the tobacco mosaic virus 126- and 183-kilodalton replicase proteins. Journal of Virology 77: 3549-3556.

Hafren A, Hofius D, Ronnholm G, et al. (2010) HSP70 and its cochaperone CPIP promote potyvirus infection in Nicotiana benthamiana by regulating viral coat protein functions. The Plant Cell 22: 523-535.

Heinlein M (2015) Plant virus replication and movement. Virology 479-480: 657-671.

Huang TS and Nagy PD (2011) Direct inhibition of tombusvirus plus-strand RNA synthesis by a dominant negative mutant of a host metabolic enzyme, glyceraldehyde-3phosphate dehydrogenase, in yeast and plants. Journal of Virology 85: 9090-9102.

Huang YW, Hu CC, Liou MR, et al. (2012) Hsp90 interacts specifically with viral RNA and differentially regulates replication initiation of Bamboo mosaic virus and associated satellite RNA. PLoS Pathogens 8: e1002726.

Hwang J, Oh CS and Kang BC (2013) Translation elongation factor 1B (eEF1B) is an essential host factor for Tobacco mosaic virus infection in plants. Virology 439: 105114. 
Hyodo K, Kaido M and Okuno T (2014) Host and viral RNA-binding proteins involved in membrane targeting, replication and intercellular movement of plant RNA virus genomes. Frontiers in Plant Science 5: 321.

Hyodo K, Mine A, Taniguchi T, et al. (2013) ADP ribosylation factor 1 plays an essential role in the replication of a plant RNA virus. Journal of Virology 87: 163-176.

Ishibashi K, Miyashita S, Katoh E and Ishikawa M (2012) Host membrane proteins involved in the replication of tobamovirus RNA. Current Opinion in Virology 2: 699-704.

Kim SH, Palukaitis P and Park YI (2002) Phosphorylation of cucumber mosaic virus RNA polymerase 2a protein inhibits formation of replicase complex. EMBO Journal 21: 2292-2300.

Kovalev N and Nagy PD (2014) The expanding functions of cellular helicases: the tombusvirus RNA replication enhancer co-opts the plant eIF4AIII-like AtRH2 and the DDX5-like AtRH5 DEAD-box RNA helicases to promote viral asymmetric RNA replication. PLoS Pathogens 10: e1004051.

Li Z, Gonzalez PA, Sasvari Z, et al. (2014) Methylation of translation elongation factor 1A by the METTL10-like See1 methyltransferase facilitates tombusvirus replication in yeast and plants. Virology 448: 43-54.

Li Z, Pogany J, Panavas T, et al. (2009) Translation elongation factor 1A is a component of the tombusvirus replicase complex and affects the stability of the p33 replication cofactor. Virology 385: 245-260.

Li Z, Pogany J, Tupman S, et al. (2010) Translation elongation factor 1A facilitates the assembly of the tombusvirus replicase and stimulates minus-strand synthesis. PLoS Pathogens 6: e1001175.

Lin JY, Mendu V, Pogany J, et al. (2012) The TPR domain in the host Cyp40-like cyclophilin binds to the viral replication protein and inhibits the assembly of the tombusviral replicase. PLoS Pathogens 8: e1002491.

Mendu V, Chiu M, Barajas D, et al. (2010) Cpr1 cyclophilin and Ess1 parvulin prolyl isomerases interact with the tombusvirus replication protein and inhibit viral replication in yeast model host. Virology 406: 342-351.

Mine A, Hyodo K, Tajima Y, et al. (2012) Differential roles of Hsp70 and Hsp90 in the assembly of the replicase complex of a positive-strand RNA plant virus. Journal of Virology 86: 12091-12104.

Nagy PD and Pogany J (2012) The dependence of viral RNA replication on co-opted host factors. Nature Reviews Microbiology 10: 137-149. 
Nagy PD, Pogany J and Lin JY (2014) How yeast can be used as a genetic platform to explore virus-host interactions: from 'omics' to functional studies. Trends in Microbiology 22: 309-316.

Nagy PD, Wang RY, Pogany J, et al. (2011) Emerging picture of host chaperone and cyclophilin roles in RNA virus replication. Virology 411: 374-382.

Newburn LR and White KA (2015) Cis-acting RNA elements in positive-strand RNA plant virus genomes. Virology 479-480: 434-443.

Nishikiori M, Sugiyama S, Xiang H, et al. (2012) Crystal structure of the superfamily 1 helicase from Tomato mosaic virus. Journal of Virology 86: 7565-7576.

Pathak KB, Pogany J and Nagy PD (2011) Non-template functions of the viral RNA in plant RNA virus replication. Current Opinion in Virology 1: 332-338.

Pathak KB, Pogany J, Xu K, et al. (2012) Defining the roles of cis-acting RNA elements in tombusvirus replicase assembly in vitro. Journal of Virology 86: 156-171.

Pathak KB, Sasvari Z and Nagy PD (2008) The host Pex19p plays a role in peroxisomal localization of tombusvirus replication proteins. Virology 379: 294-305.

Prasanth KR, Huang YW, Liou MR, et al. (2011) Glyceraldehyde 3-phosphate dehydrogenase negatively regulates the replication of Bamboo mosaic virus and its associated satellite RNA. Journal of Virology 85: 8829-8840.

Prod'homme D, Jakubiec A, Tournier V, et al. (2003) Targeting of the turnip yellow mosaic virus $66 \mathrm{~K}$ replication protein to the chloroplast envelope is mediated by the $140 \mathrm{~K}$ protein. Journal of Virology 77: 9124-9135.

Puustinen P and Mäkinen K (2004) Uridylylation of the potyvirus VPg by viral replicase NIb correlates with the nucleotide binding capacity of VPg. Journal of Biological Chemistry 279: 38103-38110.

Ray D, Na H and White KA (2004) Structural properties of a multifunctional T-shaped RNA domain that mediate efficient tomato bushy stunt virus RNA replication. Journal of Virology 78: 10490-10500.

Shah Nawaz-ul-Rehman M, Martinez-Ochoa N, Pascal H, et al. (2012) Proteome-wide overexpression of host proteins for identification of factors affecting tombusvirus RNA replication: an inhibitory role of protein kinase C. Journal of Virology 86: 93849395.

Sivakumaran K, Kim CH, Tayon R, Jr. and Kao CC (1999) RNA sequence and secondary structural determinants in a minimal viral promoter that directs replicase recognition 
and initiation of genomic plus-strand RNA synthesis. Journal of Molecular Biology 294: $667-682$.

Stork J, Kovalev N, Sasvari Z and Nagy PD (2011) RNA chaperone activity of the tombusviral p33 replication protein facilitates initiation of RNA synthesis by the viral RdRp in vitro. Virology 409: 338-347.

Tomita Y, Mizuno T, Diez J, et al. (2003) Mutation of host DnaJ homolog inhibits brome mosaic virus negative-strand RNA synthesis. Journal of Virology 77: 2990-2997.

Verchot J (2012) Cellular chaperones and folding enzymes are vital contributors to membrane bound replication and movement complexes during plant RNA virus infection. Frontiers in Plant Science 3: 275.

Wang A (2015) Dissecting the molecular network of virus-plant interactions: the complex roles of host factors. Annual Review of Phytopathology 53: 45-66.

Wang RY and Nagy PD (2008) Tomato bushy stunt virus co-opts the RNA-binding function of a host metabolic enzyme for viral genomic RNA synthesis. Cell Host Microbe 3: 178-187.

Xiong R and Wang A (2013) SCE1, the SUMO-conjugating enzyme in plants that interacts with NIb, the RNA-dependent RNA polymerase of Turnip mosaic virus, is required for viral infection. Journal of Virology 87: 4704-4715.

Xu K and Nagy PD (2014) Expanding use of multi-origin subcellular membranes by positivestrand RNA viruses during replication. Current Opinion in Virology 9: 119-126.

\section{Further Reading}

Hyodo K and Okuno T (2016) Pathogenesis mediated by proviral host factors involved in translation and replication of plant positive-strand RNA viruses. Current Opinion in Virology 17: 11-18.

Mine A and Okuno T (2012) Composition of plant virus RNA replicase complexes. Current Opinion in Virology 2: 669-675.

Nagy PD (2015) Viral sensing of the subcellular environment regulates the assembly of new viral replicase complexes during the course of infection. Journal of Virology 89: 51965199.

Rao AL, Chaturvedi S and Garmann RF (2014) Integration of replication and assembly of infectious virions in plant RNA viruses. Current Opinion in Virology 9: 61-66. 
Saxena P and Lomonossoff GP (2014) Virus infection cycle events coupled to RNA replication. Annual Review of Phytopathology 52: 197-212. 
Tables and Figures

Table 1. Plant RNA virus families and genera and important properties.

\begin{tabular}{|c|c|c|c|c|c|}
\hline Genome & Family & Genus & $\begin{array}{c}\text { Type } \\
\text { species }^{\mathrm{a}}\end{array}$ & $\begin{array}{c}\text { Number of } \\
\text { segments }\end{array}$ & $\begin{array}{c}\text { Virion } \\
\text { morphology }\end{array}$ \\
\hline \multirow[t]{7}{*}{ ssRNA(-) } & Bunyaviridae $^{a}$ & Tospovirus & TSWV & 3 & Spherical, enveloped \\
\hline & Ophioviridae & Ophiovirus & CSV & $3-4$ & Filamentous \\
\hline & Rhabdoviridae $^{b}$ & Cytorhabdovirus & LNYV & 1 & Bacilliform, enveloped \\
\hline & & Nucleorhabdovirus & PYDV & 1 & Bacilliform, enveloped \\
\hline & Unassigned & Emaravirus & EMAAV & 4 & Spherical, enveloped \\
\hline & & Tenuivirus & RSV & 4 & Filamentous \\
\hline & & Varicosavirus & LBVaV & 2 & Rodlike \\
\hline \multirow[t]{50}{*}{$\operatorname{ssRNA}(+)$} & Alphaflexiviridae ${ }^{c}$ & Allexivirus & ShVX & 1 & Filamentous \\
\hline & & Lolavirus & LoLV & 1 & Filamentous \\
\hline & & Mandarivirus & ICRSV & 1 & Filamentous \\
\hline & & Potexvirus & PVX & 1 & Filamentous \\
\hline & Benyviridae & Benyvirus & BNYVV & 5 & Rodlike \\
\hline & Betaflexiviridae $^{c}$ & Capillovirus & ASGV & 1 & Filamentous \\
\hline & & Carlavirus & CLV & 1 & Filamentous \\
\hline & & Citrivirus & CLBV & 1 & Filamentous \\
\hline & & Foveavirus & ASPV & 1 & Filamentous \\
\hline & & Tepovirus & PVT & 1 & Filamentous \\
\hline & & Trichovirus & ACLSV & 1 & Filamentous \\
\hline & & Vitivirus & GVA & 1 & Filamentous \\
\hline & Bromoviridae & Alfamovirus & AMV & 3 & Bacilliform \\
\hline & & Anulavirus & PZSV & 3 & Isometric \\
\hline & & Bromovirus & BMV & 3 & Isometric \\
\hline & & Cucumovirus & $\mathrm{CMV}$ & 3 & Isometric \\
\hline & & Ilarvirus & TSV & 3 & Isometric \\
\hline & & Oleavirus & OLV-2 & 3 & Isometric \\
\hline & Closteroviridae & Ampelovirus & GLRaV-3 & 1 & Filamentous \\
\hline & & Closterovirus & BYV & 1 & Filamentous \\
\hline & & Crinivirus & LIYV & 2 & Filamentous \\
\hline & & Velarivirus & GLRaV-7 & 1 & Filamentous \\
\hline & Comovirinae $^{d}$ & Comovirus & CPMV & 2 & Isometric \\
\hline & & Fabavirus & BBWV-1 & 2 & Isometric \\
\hline & & Nepovirus & TRSV & 2 & Isometric \\
\hline & Luteoviridae & Enamovirus & PEMV-1 & 1 & Isometric \\
\hline & & Luteovirus & BYDV-PAV & 1 & Isometric \\
\hline & & Polerovirus & PLRV & 1 & Isometric \\
\hline & Marnaviridae $^{d}$ & Marnavirus & HaRNAV & 1 & Isometric \\
\hline & Potyviridae & Brambyvirus & BVY & 1 & Filamentous \\
\hline & & Bymovirus & BaYMV & 1 & Filamentous \\
\hline & & Ipomovirus & SPMMV & $1-2$ & Filamentous \\
\hline & & Macluravirus & MacMV & $1-2$ & Filamentous \\
\hline & & Poacevirus & TriMV & 1 & Filamentous \\
\hline & & Potyvirus & PVY & 1 & Filamentous \\
\hline & & Rymovirus & RGMV & $1-2$ & Filamentous \\
\hline & & Tritimovirus & WSMV & $1-2$ & Filamentous \\
\hline & Secoviridae ${ }^{d}$ & Cheravirus & CRLV & 2 & Isometric \\
\hline & & Sadwavirus & SDV & 2 & Isometric \\
\hline & & Sequivirus & PYFV & 1 & Isometric \\
\hline & & Torradovirus & ToTV & 2 & Isometric \\
\hline & & Waikavirus & RTSV & 1 & Isometric \\
\hline & Tombusviridae & Alphanecrovirus & TNV-A & 1 & Isometric \\
\hline & & Aureusvirus & PoLV & 1 & Isometric \\
\hline & & Avenavirus & OCSV & 1 & Isometric \\
\hline & & Betanecrovirus & TNV-D & 1 & Isometric \\
\hline & & Carmovirus & CarMV & 1 & Isometric \\
\hline & & Dianthovirus & CRSV & 2 & Isometric \\
\hline & & Gallantivirus & GMV & 1 & Isometric \\
\hline & & Macanavirus & FNSV & 1 & Isometric \\
\hline
\end{tabular}




\begin{tabular}{|c|c|c|c|c|c|}
\hline & & Machlomovirus & MDMV & 1 & Isometric \\
\hline & & Panicovirus & PMV & 1 & Isometric \\
\hline & & Tombusvirus & TBSV & 1 & Isometric \\
\hline & & Umbravirus & $\mathrm{CMOV}^{\mathrm{e}}$ & 1 & Isometric \\
\hline & & Zeavirus & $\mathrm{MNeSV}$ & 1 & Isometric \\
\hline & Tymoviridae & Maculavirus & $\mathrm{GFkV}$ & 1 & Isometric \\
\hline & & Marafivirus & MRFV & 1 & Isometric \\
\hline & & Tymovirus & TYMV & 1 & Isometric \\
\hline & Unassigned & Cilevirus & CiLV-C & 2 & Bacilliform \\
\hline & & Higrevirus & HGSV-2 & 3 & Baciliform \\
\hline & & Idaeovirus & RBDV & 3 & Isometric \\
\hline & & Ourmiavirus & OuMV & 3 & Isometric \\
\hline & & Polemovirus & PnLV & 1 & Isometric \\
\hline & & Sobemovirus & SBMV & 1 & Isometric \\
\hline & Virgaviridae & Furovirus & SBWMV & 2 & Rodlike \\
\hline & & Hordeivirus & BSMV & $3-4$ & Rodlike \\
\hline & & Pecluvirus & PCV & 2 & Rodlike \\
\hline & & Pomovirus & PMTV & 3 & Rodlike \\
\hline & & Tobamovirus & TMV & 1 & Rodlike, \\
\hline & & Tobravirus & TRV & 2 & Rodlike \\
\hline & & Amalgavirus & STV & 1 & Unknown \\
\hline & Amalgaviridae & Endornavirus & VFV & 1 & Unknown \\
\hline & Endornaviridae & Alphapartitivirus & WCCV-1 & 2 & Isometric \\
\hline & Partitiviridae & Betapartitivirus & WCCV-2 & 2 & Isometric \\
\hline & & Deltapartitivirus & PCV-1 & 2 & Isometric \\
\hline & & Fijivirus & FDV & 10 & Isometric \\
\hline & Reoviridae & Oryzavirus & RRSV & 10 & Isometric \\
\hline & & Phytoreovirus & WTV & 12 & Isometric \\
\hline
\end{tabular}

a Abbreviations: ACLSV, Apple chlorotic leaf spot virus; AMV, Alfalfa mosaic virus; ASGV, Apple stem grooving virus; ASPV, Apple stem pitting virus; BaYMV, Barley yellow mosaic virus; BBWV-1, Broad bean wilt virus 1; BMV, Brome mosaic virus; BNYVV, Beet necrotic yellow vein virus; BSMV, Barley stripe mosaic virus; BVY, Blackberry virus Y; BYDVPAV, Barley yellow dwarf virus-PAV; BYV, Beet yellow virus; CarMV, Carnation mottle virus; CiLV-C, Citrus leprosis virus $C$; CLBV, Citrus leaf blotch virus; CLV, Carnation latent virus; CMoV, Carrot mottle virus; CMV, Cucumber mosaic virus; CPMV, Cowpea mosaic virus; CRLV, Cherry rasp leaf virus; CRSV, Carnation ringspot virus; CSV, Citrus psorosis virus; EMAAV, European mountain ash ringspot-associated virus; FDV, Fiji disease virus; FNSV, Furcraea necrotic streak virus; GFkV, Grapevine fleck virus; GLRaV-3/7, Grapevine leafroll-associated virus 3/7; GMV, Galinsoga mosaic virus; GVA, Grapevine virus A; HaRNAV, Heterosigma akashiwo RNA virus; HGSV-2, Hibiscus green spot virus 2; ICRSV, Indian citrus ringspot virus; LBVaV, Lettuce big-vein virus; LIYV, Lettuce infectious yellows virus; LoLV, Lolium latent virus; LNYV, Lettuce necrotic yellows virus; MacMV, Maclura mosaic virus; MCMV, Maize chlorotic mottle virus; MNeSV, Maize necrotic streak virus; MRFV, Maize rayado fino virus; OCSV, Oat chlorotic stunt virus; OLV-2, Olive latent virus; OuMV, Ourmia melon virus; PaSV, Pangola stunt virus; PCV, Peanut clump virus; PCV-1, Pepper cryptic virus 1; PEMV-1, Pea enation mosaic virus-1; PLRV, Potato leafroll virus; PMTV, Potato mop-top virus; PMV, Panicum mosaic virus; PnLV, Poinsettia latent virus; PoLV, Pothos latent virus; PVT/X/Y, Potato virus T/X/Y; PYDV, Potato yellow dwarf virus; PYFV, Parsnip yellow fleck virus; PZSV, Pelargonium zonate spot virus; RBDV, Raspberry bushy dwarf virus; RGMV, Ryegrass mosaic virus; RRSV, Rice ragged stunt virus; RSV, Rice stripe virus; RTSV, Rice tungro spherical virus; SBMV, Southern bean mosaic virus; SBWMV, Soil-borne wheat mosaic virus; SDV, Satsuma dwarf virus; ShVX, Shallot virus X; SPMMV, Sweet potato mild mottle virus; STV, Southern tomato virus; TBSV, Tomato bushy stunt virus; Tobacco mosaic virus; TNV-A/D, Tobacco necrosis virus A/D; ToTV, Tomato torrado virus; TriMV, Triticum mosaic virus; TRSV, Tobacco ringspot virus; TRV, Tobacco rattle virus; TSV, Tobacco streak virus; TSWV, Tomato spotted wilt virus; TYMV, Turnip yellow mosaic virus; VFV, Vicia faba endornavirus; WCCV-1/2, White clover cryptic virus 1/2; WSMV, Wheat streak mosaic virus; WTV, Wound tumor virus.

${ }^{\mathrm{b}}$ Order Mononegavirales

${ }^{\mathrm{c} O r d e r}$ Tymovirales

${ }^{\mathrm{d}}$ Order Picornavirales

${ }^{\mathrm{e}}$ Satellite virus 
Table 2. Site of replication of different plant RNA viruses.

\begin{tabular}{|c|c|c|c|}
\hline Family & Genus & Virus & Membranes used for replication \\
\hline \multirow[t]{4}{*}{ Bromoviridae } & Alfamovirus & Alfalfa mosaic virus (AMV) & Vacuolar membrane \\
\hline & Bromovirus & Brome mosaic virus (BMV) & Endoplasmic reticulum \\
\hline & Cucumovirus & Cucumber mosaic virus (CMV) & Vacuolar membrane \\
\hline & Cucumovirus & Tomato aspermy virus (TAV) & Vacuolar membrane \\
\hline \multirow[t]{2}{*}{ Potyviridae } & Potyvirus & Plum pox virus (PPV) & Endoplasmic reticulum \\
\hline & & Tobacco etch virus (TEV) & Endoplasmic reticulum \\
\hline \multirow[t]{3}{*}{ Rhabdoviridae } & Cytorhabdovirus & Lettuce necrotic yellows virus (LNYV) & Endoplasmic reticulum \\
\hline & Nucleorhabdovirus & Potato yellow dwarf virus (PYDV) & Nuclear membrane \\
\hline & & Sonchus yellow net virus (SYNV) & Nuclear membrane \\
\hline \multirow[t]{2}{*}{ Secoviridae } & Comovirus & Cowpea mosaic virus (CPMV) & Endoplasmic reticulum \\
\hline & Nepovirus & Grapevine fanleaf virus (GFL) & Endoplasmic reticulum \\
\hline \multirow[t]{8}{*}{ Tombusviridae } & Carmovirus & Melon necrotic spot virus (MNSV) & Mitochondria \\
\hline & & Pelargonium flower break virus (PFBV) & Mitochondria \\
\hline & Dianthovirus & Red clover necrotic mosaic virus (RCNMV) & Endoplasmic reticulum \\
\hline & Tombusvirus & Carnation Italian ringspot virus (CIRV) & Mitochondria \\
\hline & & Cucumber necrosis virus (CNV) & Peroxisome \\
\hline & & Cymbidium ringspot virus (CymRSV) & Peroxisome \\
\hline & & Tomato bushy stunt virus (TBSV) & Peroxisome \\
\hline & Umbravirus & Lettuce speckles mottle virus (LSMV) & Vacuolar membrane \\
\hline Tymoviridae & Tymovirus & Turnip yellow mosaic virus (TUMV) & Chloroplast outer membrane \\
\hline Unassigned & Benyvirus & Beet necrotic yellow vein virus (BNYVV) & Mitochondria \\
\hline \multirow[t]{4}{*}{ Virgaviridae } & Pecluvirus & Peanut clump virus (PCV) & Endoplasmic reticulum \\
\hline & Tobamovirus & Cucumber green mottle mosaic virus (CGMMV) & Mitochondria \\
\hline & & Tobacco mosaic virus (TMV) & Endoplasmic reticulum \\
\hline & Tobravirus & Tobacco rattle virus (TRV) & Mitochondria \\
\hline
\end{tabular}


Table 3. Cellular factors involved in plant virus RNA replication.

\begin{tabular}{|c|c|c|c|}
\hline Host Factor $^{\mathrm{a}}$ & Cellular function & Function in viral replication & Virus $^{b}$ \\
\hline ACBP & Membrane synthesis & Lipid synthesis & BMV \\
\hline ARF1 & Membrane trafficking & $\begin{array}{l}\text { COPI vesicle formation, remodeling } \\
\text { of ER membranes }\end{array}$ & RCNMV \\
\hline $\begin{array}{l}\text { ARL8, TOM1, } \\
\text { TOM2A, TOM3 }\end{array}$ & Integral membrane protein & $\begin{array}{l}\text { VRC assembly, enhance VRC RNA } \\
\text { synthesizing and capping functions }\end{array}$ & ToMV \\
\hline $\begin{array}{l}\text { AtRH2, AtRH5, } \\
\text { AtRH8, RH20 }\end{array}$ & DEAD-box RNA Helicases & $\begin{array}{l}\text { Colocalizes with VRC, facilitates } \\
\text { asymmetric RNA replication }\end{array}$ & TBSV, TuMV \\
\hline BRO1, DOA4 & Membrane synthesis & Lipid metabolism & BMV \\
\hline $\mathrm{CDC} 34$ & $\begin{array}{l}\text { E2 Ubiquitin conjugating } \\
\text { enzyme }\end{array}$ & $\begin{array}{l}\text { Ubiquitination of a viral RNA } \\
\text { replication protein }\end{array}$ & TBSV \\
\hline $\begin{array}{l}\text { CHO1, INO1, } \\
\text { INO2, INO4, } \\
\text { OPI3 }\end{array}$ & Membrane synthesis & $\begin{array}{l}\text { Phospholipid homeostasis required } \\
\text { for activation or assembly of VRC }\end{array}$ & TBSV \\
\hline CPIP & Co-chaperone & Regulation of coat protein functions & PVA \\
\hline CIPK12 & Phosphorylation & $\begin{array}{l}\text { Phosphorylation of viral RNA } \\
\text { polymerase to inhibit replication }\end{array}$ & CMV \\
\hline CPR1, CPR7 & Chaperone & Initiation of RNA replication & TBSV \\
\hline $\begin{array}{l}\text { DED1, DBP2, } \\
\text { RH20 }\end{array}$ & RNA-binding protein & Synthesis of RNA(+) & BMV, TBSV \\
\hline eEF1A & RNA-binding protein & Synthesis of RNA (+) or (-) strand & $\begin{array}{l}\text { TBSV, TMV, TSWV, } \\
\text { TuMV, TYMV }\end{array}$ \\
\hline eEF1B & RNA-binding protein & Interaction with VRC & TMV \\
\hline eEF1B $B_{Y}$ & RNA-binding protein & Synthesis of RNA (-) strand & TBSV \\
\hline eIF3 & RNA-binding protein & Synthesis of RNA (-) strand & BMV \\
\hline $\begin{array}{l}\text { ERG25, SMO1, } \\
\text { SMO2 }\end{array}$ & Membrane synthesis & $\begin{array}{l}\text { Sterol biosynthesis required for } \\
\text { activation or assembly of VRC }\end{array}$ & Tombusvirus \\
\hline ESCRT & Membrane trafficking & $\begin{array}{l}\text { Remodeling membranes for } \\
\text { activation or assembly of VRC }\end{array}$ & BMV, CIRV, TBSV \\
\hline GAPDH & $\begin{array}{l}\text { Cytosolic energy } \\
\text { production }\end{array}$ & $\begin{array}{l}\text { VRC assembly or inhibition of RNA } \\
\text { replication }\end{array}$ & $\begin{array}{l}\text { BaMV, CNV, RCNMV, } \\
\text { TBSV }\end{array}$ \\
\hline HSF1 & $\begin{array}{l}\text { Trimeric heat shock } \\
\text { transcription factor }\end{array}$ & $\begin{array}{l}\text { Modulation of RNA polymerase } \\
\text { levels or localization }\end{array}$ & BMV \\
\hline HSP70, HSP90 & Chaperone & VRC assembly or activation & $\begin{array}{l}\text { BaMV, BYV, CNV, CTV, } \\
\text { PVA, RCNMV, TBSV, } \\
\text { TMV, ToMV, TuMV }\end{array}$ \\
\hline $\begin{array}{l}\text { LSm1- } \\
\text { 7/Dhh1p/Pat1p }\end{array}$ & mRNA decay & VRC assembly & BMV \\
\hline OLE1 & Membrane synthesis & Fatty acids synthesis & BMV \\
\hline OPI1P & Membrane synthesis & $\begin{array}{l}\text { Transcriptional repressor of } \\
\text { phospholipid synthesis in yeast }\end{array}$ & Tombusvirus \\
\hline ORD, VAP & Membrane synthesis & Biogenesis of replication organelles & TBSV \\
\hline OSBP & Membrane synthesis & Sterol channeling to replication sites & TBSV \\
\hline PABP & RNA-binding protein & VRC assembly & Potyvirus \\
\hline PAP85 & Seed storage protein & VRC assembly & TMV \\
\hline $\mathrm{PE}$ & Membrane synthesis & VRC assembly & TBSV \\
\hline PEX19 & Cellular membrane related & VRC assembly & TBSV \\
\hline PGK & Glycolysis & Synthesis of RNA (-) strand & BaMV \\
\hline $\mathrm{PKC1}$ & Phosphorylation & $\begin{array}{l}\text { Phosphorylation of viral protein to } \\
\text { inhibit replication }\end{array}$ & TBSV \\
\hline PLD & Membrane synthesis & VRC assembly & BMV, RCNMV \\
\hline PRE1, RPT6 & Proteasome components & $\begin{array}{l}\text { Modulation of RNA polymerase } \\
\text { levels or localization }\end{array}$ & BMV \\
\hline RHPs & Membrane trafficking & Membrane rearrangements & BMV \\
\hline Rpb11 & Transcription factor & $\begin{array}{l}\text { Regulation of viral replication } \\
\text { proteins accumulation }\end{array}$ & Tombusvirus \\
\hline RPN11 & Proteasome component & VRC assembly & Tombusvirus \\
\hline RSP5 & Ubiquitin ligase & $\begin{array}{l}\text { Cell intrinsic restriction factor } \\
\text { regulating viral replication }\end{array}$ & Tombusvirus \\
\hline SAR1 & Membrane trafficking & $\begin{array}{l}\text { COPII vesicle formation, } \\
\text { remodeling of ER membranes }\end{array}$ & RCNMV, WYMV \\
\hline
\end{tabular}




\begin{tabular}{|l|l|l|l|}
\hline SCE1 & SUMOylation & $\begin{array}{l}\text { Interaction with viral RdRP to } \\
\text { inhibit replication }\end{array}$ & TuMV \\
\hline SEC39 & Membrane trafficking & $\begin{array}{l}\text { Recognizes COPI vesicles and } \\
\text { functions in peroxisome biogenesis }\end{array}$ & TBSV \\
\hline SEE1 & Methyltransferase & eEF1A methylation & TBSV \\
\hline SKP1 & Chaperone & $\begin{array}{l}\text { Favors viral asymmetric RNA } \\
\text { replication }\end{array}$ & Nanovirus, PVX \\
\hline STI1P & Chaperone & $\begin{array}{l}\text { Cell intrinsic restriction factor } \\
\text { regulating viral replication }\end{array}$ & CIRV \\
\hline SYP71 & Membrane trafficking & Traffic to chloroplasts & TuMV \\
\hline TIP1, TIP2 & Aquaporins & Association of VRC to the tonoplast & CMV \\
\hline YDJ1 & Chaperone & VRC assembly & BMV \\
\hline
\end{tabular}

aACBP, Acyl-CoA-binding protein; ARF1, ADP ribosylation factor 1, GTPase; ARL8 GTPase; BRO1 and DOA4, components of the multivesicular body secretory pathway; CDC34, ubiquitin conjugating enzyme; CHO1, phospholipid synthesis protein; CIPK12, calcineurin B-like calcium sensor-interacting protein kinase 12; CPIP, co-chaperone of HSP70; CPR1, CPR7: cyclophilins; DED1, DPB2 and 7/Dhh1p/Pat1p, protein complexes promoting mRNA degradation; eEF1A, translation elongation factor; eEF1B 1 , translation elongation factor; eIF3, translation initiation factor; ERG25, SMO1 and SMO2, sterol biosynthesis proteins; ESCRT, endosomal sorting complexes required for transport, transport of endosomal proteins; GAPDH, glyceraldehyde 3-phosphate dehydrogenase; HSF1, trimeric heat shock transcription factor 1; HSP70 and HSP90, heat-shock proteins; INO1 and INO2, phospholipid synthesis proteins; LSm1-7/Dhh1p/Pat1p, protein complexes promoting mRNA degradation; OLE1, unsaturated fatty acid $\Delta 9$; OPI1P, FFAT-motif (two phenylalanines with acidic tail)containing protein; OPI3, phospholipid synthesis protein; OSBP, oxysterol binding protein; PABP, Poli(A)-binding protein; PAP85, vicilin-like seed storage protein; PE, phosphatidylethanolamine; PEX19, cellular transporter; PGK, phosphoglicerate kinase; PKC1, protein kinase C1; PLD, phosphatidic acid-generating enzyme, phospholipase D; PRE1, beta 4 subunit of the $20 \mathrm{~S}$ core of the $26 \mathrm{~S}$ proteasome; RHPs, proteins homologs to the reticulum; RPN11, 26S proteasome non-ATPase subunit; RPT6, ATPase 6 of the 19S regulatory particle of the 26S proteasome; RSP5, ubiquitin ligase; SAR1, secretion-associated RAS-related 1; SCE1, SUMO-conjugating E2 enzyme; SEC39, vesicle-mediated transport protein; SEE1, METTL10-like methyltransferase; SKP1, S-phase kinase-associated protein 1; STI1P, Hop-like stress-inducible protein 1; SYP71, plantspecific Qc-SNARE protein; TIP1 and TIP2, tonoplast intrinsic proteins 1 and 2; TOM1 and TOM3, transmembrane proteins; VAP, VAMP-associated protein; YDJ1, protein of the family of heat-shock proteins HSP40.

${ }^{\mathrm{b}} \mathrm{BaMV}$, Bamboo mosaic virus; BMV, Brome mosaic virus; BYV, Beet yellow virus; CMV, Cucumber mosaic virus; CNV, Cucumber necrotic virus; CIRV, Carnation italian ringspot virus; CTV, Citrus tristeza virus; RCNMV, Red clover necrotic mosaic virus; ToMV, Tomato mosaic virus; TBSV, Tomato bushy stunt virus; TMV, Tobacco mosaic virus; TSWV, Tomato spotted wilt virus; TuMV, Turnip mosaic virus; PVA, Potato virus A; PVX, Potato virus X; TSWV, Tomato spotted wilt virus; TYMV, Turnip yellow mosaic virus; WYMV, Wheat yellow mosaic virus. 
(+) RNA virus

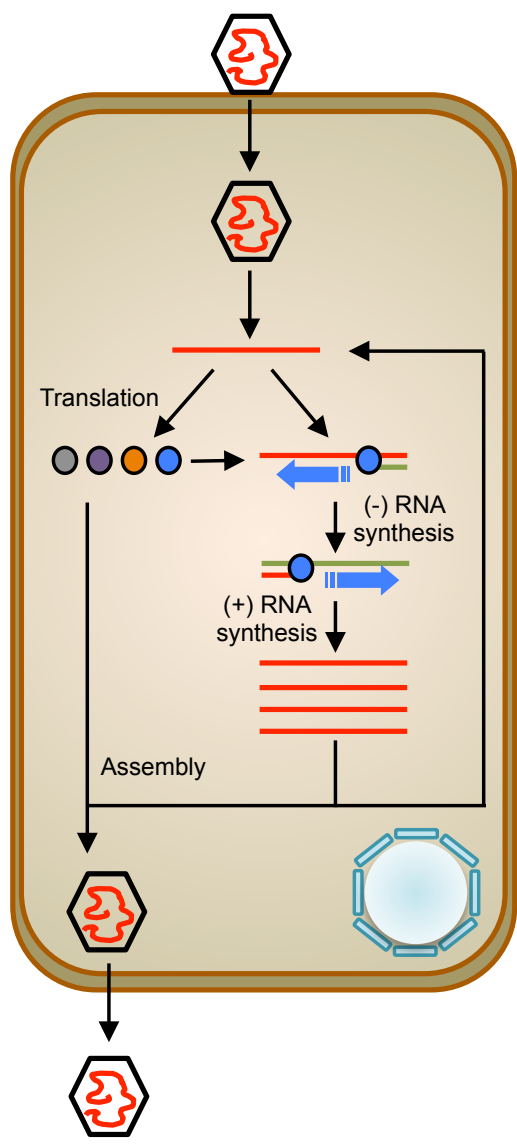

(-) RNA virus

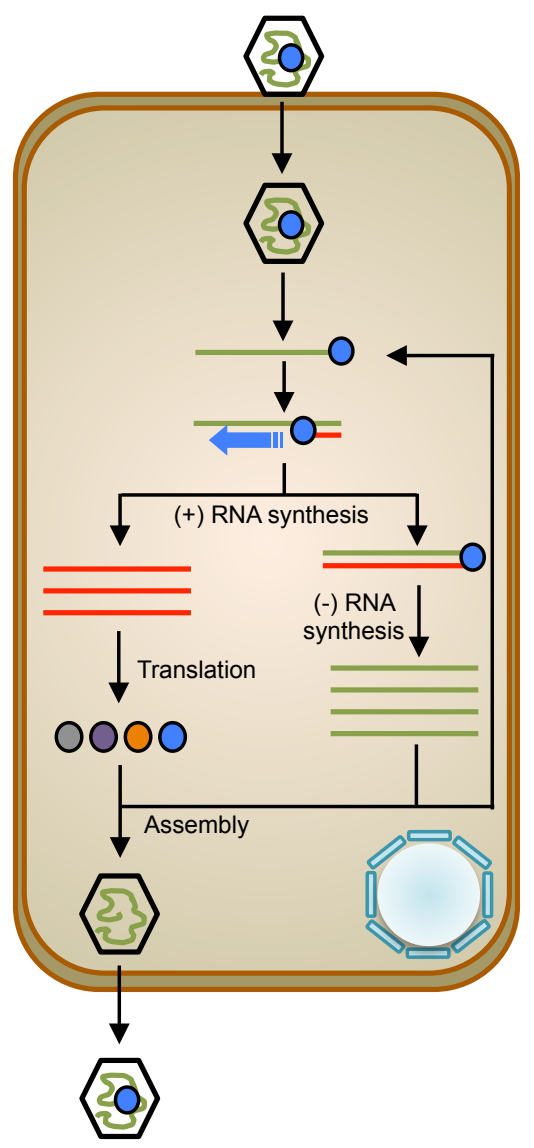

dsRNA virus

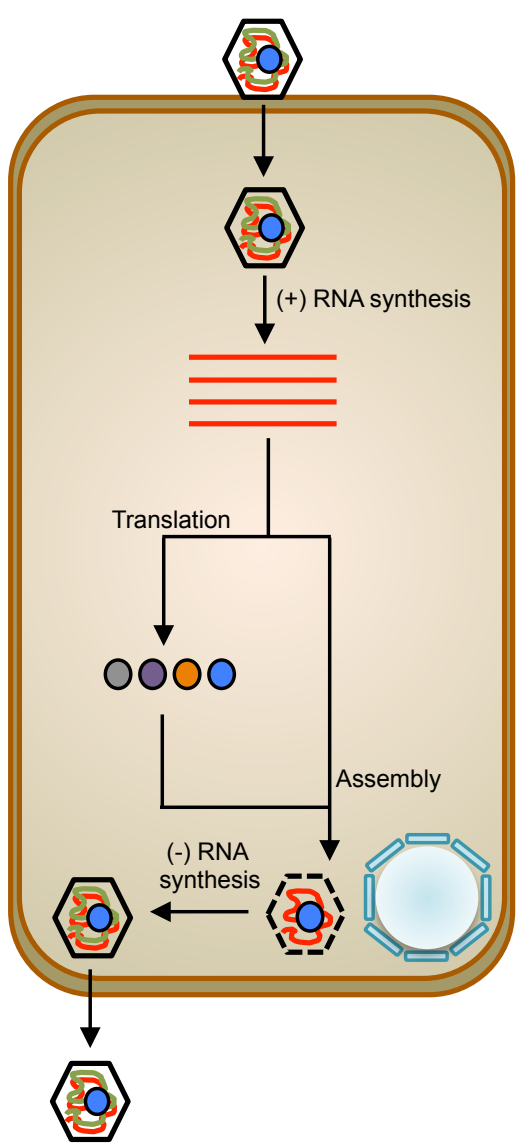

Figure 1. Cellular life cycles of plant RNA viruses. For each class of RNA virus, a simplified and representative life cycle is shown. All three virus classes replicate exclusively through RNA intermediates in the cytoplasm. Nuclear trafficking of macromolecules during viral replication could not be ruled out. Virions are shown as black hexagons containing positive $(+)$ and/or negative (-) RNA strands in red and green, respectively, and viral RNA-dependent RNA polymerase (RdRp, blue circle). Other viral proteins are shown as filled circles of distinct colors. Left: life cycle of $(+)$ RNA viruses. The genomic RNA (gRNA) from virions is released into the cytoplasm. This $(+)$ RNA, mRNA sense, is translated to produce several viral proteins including the viral RdRp. This polymerase copies the $(+)$ strands into (-) strands that serve as templates for the synthesis of $(+)$ strands that can be translated, replicated or assembled in virion particles. Middle, life cycle of the majority of (-) RNA viruses. The gRNA is used as template by the viral RdRp to generate $(+)$ strand mRNAs that are released into the cytoplasm to be translated into viral proteins. The gRNA is also copied into full-length $(+)$ strands that in turn serve as templates for (-) RNA synthesis. For members of the genus Nucleorhabdovirus replication occurs in the nucleus. Right, life cycle of double-stranded RNA (dsRNA) viruses. Viral RdRp synthesizes and releases into the cytoplasm $(+)$ strands that are first translated and 
then packaged to form immature virions. Virions mature by synthesizing (-) RNA and by the addition of other viral proteins. 


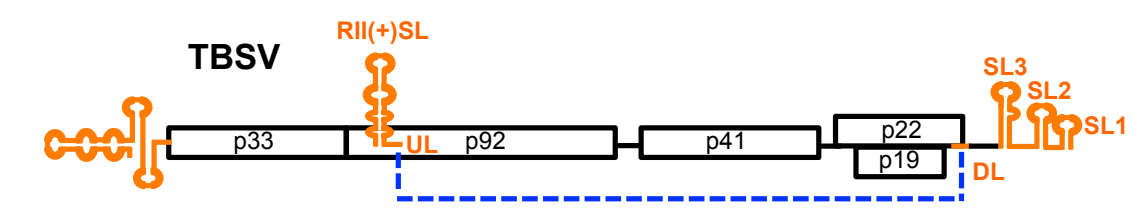

(a)

(b)

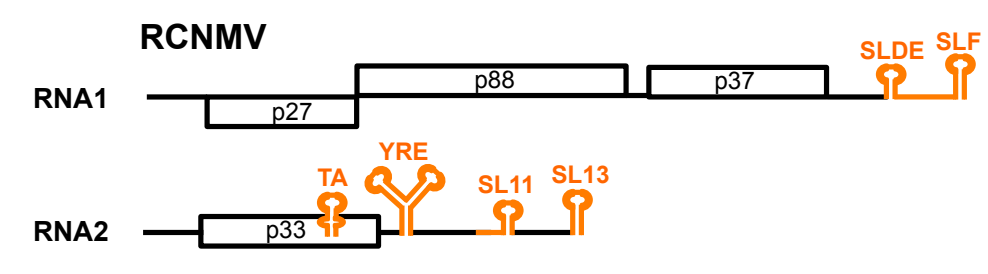

\section{BMV}

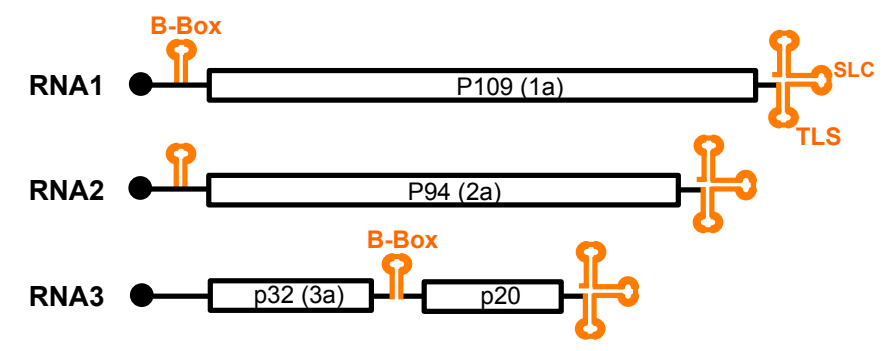

(c)

Figure 2. Cis-acting RNA elements in viral genomic RNAs (gRNAs) involved in genome replication. A diagram corresponding to the linear RNA genome is shown for each selected virus described in the main text. RNA elements in the viral RNA genome involved in replication are shown in orange, and the long-range interactions between them are shown in blue dotted lines. (a) RNA elements in Tomato bushy stunt virus (TBSV) gRNA. (b) RNA elements in Red clover necrotic mosaic virus (RCNMV) gRNAs. (c) RNA elements in Brome mosaic virus (BMV) gRNAs. Adapted from Newburn and White (2015) C Elsevier. 